\title{
Effet du numéro de lactation sur l'aptitude à la coagulation du lait de vache
}

\author{
JB Coulon 1, D Machebœuf 2 \\ 1 INRA, laboratoire adaptation des herbivores aux milieux, Theix, 63122 Saint-Genès-Champanelle; \\ 2 INRA, laboratoire de recherches fromagères, route de Salers, 15000 Aurillac, France
}

(Reçu le 21 mai 1993; accepté le 13 septembre 1993)

\begin{abstract}
Résumé - Sur 20 vaches laitières de races Holstein, Montbéliarde et Tarine, conduites de manière semblable au cours de leur 2 premières lactations, 5 échantillons de lait ont été prélevés au cours de chaque lactation ( 3 au cours de la période hivernale et 2 au pâturage). La composition chimique du lait ainsi que son aptitude à la coagulation (avec un appareil Formagraph) ont été mesurées. Lorsque l'on tient compte du taux de caséines, les différents paramètres de la coagulation du lait sont améliorés de 20 à $30 \%$ en deuxième lactation. Les différentes causes de cet écart sont discutées.
\end{abstract}

vache laitière / numéro de lactation / coagulation du lait

Summary - Effect of lactation number on cows' milk coagulation properties. Twenty dairy cows ( 7 Holstein, 4 Montbéliarde, and 9 Tarine) were managed identically during their first 2 lactations. During each lactation milk samples were collected from the 20 cows, 3 times in the winter and twice during the grazing period. The chemical composition and coagulation properties (measured with a Formagraph) of the milk were determined. When the casein content was taken into account, the coagulation characteristics were better in the second lactation. The differences ranged from 20 to $30 \%$ according to the characteristics. The possible origins of these differences are discussed.

dairy cow / lactation number / milk coagulation

\section{INTRODUCTION}

L'aptitude du lait à la coagulation est un des facteurs déterminants de la quantité de fromage produite et de sa qualité. Elle dépend à la fois des caractéristiques génétiques (race et/ou variants génétiques des protéines) (Grosclaude, 1988) et physiologiques des animaux (stade de lacta- tion) (Coulon et al, 1991) et de celles de l'alimentation quand celle-ci affecte la composition chimique du lait, et en particulier le taux protéique (Machebœuf et al, 1993). L'objectif de cette étude a été de préciser, sur des animaux conduits de manière identique pendant 2 lactations consécutives, l'effet du numéro de lactation sur les caractéristiques rhéologiques du lait. 


\section{MATÉRIEL ET MÉTHODES}

\section{Dispositif expérimental}

Vingt vaches de races Holstein (7), Montbéliarde (4) ou Tarine (9), ayant vêlé entre la fin novembre et la fin janvier ont été utilisées. Elles ont reçu, au cours de leurs 2 premières lactations hivernales, une ration composée de foin de prairie naturelle, offert à volonté, et complémenté à l'aide d'un aliment concentré équilibré, à un niveau haut ou bas selon les animaux. $\mathrm{Ce}$ niveau a été le même au cours des 2 hivers consécutifs pour une vache donnée. Ces vaches ont ensuite été conduites ensemble au pâturage. Les conditions de cet essai ont été décrites en détail par Machebœuf et al (1993).

\section{Mesures et analyses}

Des échantillons de lait ont été recueillis individuellement à la traite du matin, 3 fois au cours de l'hiver (1:février, 2:mars et 3:avril) et 2 fois au cours de la période de pâturage (4:juin et 5:juillet). Sur chacun de ces échantillons, des mesures de composition chimique et d'aptitude à la coagulation ont été réalisées.

Les teneurs en lactose, en calcium, en matières grasses, et en matières azotées totales ont été déterminées, ainsi que les différentes fractions azotées du lait (caséines, protéines solubles et urée) (cf Machebœuf et al, 1993). Par ailleurs, une numération cellulaire du lait a été effectuée, ainsi qu'un contrôle bactériologique (flore totale et contamination butyrique).

Le $\mathrm{pH}$ du lait a été mesuré juste après la traite. Les mesures rhéologiques ont été effectuées à l'aide d'un Formagraph selon la méthode proposée par Mc Mahon et Brown (1982). Les mesures ont été réalisées au $\mathrm{pH}$ initial du lait, puis après standardisation du $\mathrm{pH}$ à une valeur de 6,60 (par adjonction de quelques gouttes d'acide lactique $0.1 \mathrm{~N}$ ). Toutes les mesures ont été réalisèes en double. Les paramètres rhéologiques mesurés ont été le temps de coagulation $(R)$, le temps de raffermissement nécessaire pour obtenir une fermeté de $20 \mathrm{~mm}$ (K20) et la fermeté du gel en fin d'analyse (A30). Des mesures effectuées sur une poudre de lait reconstituée ont permis de contrôler que la déviation de la méthode au cours du temps était négligeable (les valeurs moyennes observées sur les laits témoins au cours des 2 années ont été de respectivement 3,6 et $3,4 \mathrm{~min}$ pour le temps de coagulation, 2,0 et $2,1 \mathrm{~min}$ pour le temps de raffermissement et 61,2 et $60,5 \mathrm{~mm}$ pour la fermeté du gel. Pour ces 3 paramètres, les coefficients de variation ont été respectivement de $2,5,4,5$ et $1,1 \%$ ). Les mesures réalisées à $\mathrm{pH}$ standardisé ayant conduit aux mêmes différences entre traitements que celles obtenues au pH initial du lait, seuls ces derniers résultats seront détaillés.

L'effet du numéro de lactation a été étudié sur les moyennes hivernales et estivales. Pour chacune de ces 2 périodes, les données ont été traitées par analyse de variance. Les facteurs introduits ont été le numéro de lactation et la race des animaux.

\section{RÉSULTATS}

La comparaison des valeurs obtenues au cours des 2 hivers ne met en évidence aucune différence entre la première et la seconde lactation des animaux sur les différents critères d'aptitude à la coagulation (tableau I). La teneur en caséines du lait a cependant été inférieure de $1,4 \mathrm{~g} / \mathrm{l}(P<$ $0,05)$ au cours du $2^{e}$ hiver. À l'inverse, au cours de la période estivale, les valeurs de l'ensemble des caractéristiques rhéologiques ont été améliorées en seconde lactation (de 20 à $30 \%$ selon les paramètres) (tableau I), bien que les taux de caséines aient été identiques les 2 années. Seul le taux de protéines solubles a été supérieur la seconde année $(+1 \mathrm{~g} / \mathrm{l} ; P<0,01)$. Quelle que soit la période, les taux de calcium du lait ont été significativement plus élevés chez les vaches en seconde lactation que chez les primipares $(+0,17 \mathrm{~g} / \mathrm{l}$; $P<0,01)$.

D'une lactation à l'autre, les caractéristiques rhéologiques des laits ont été très répétables (fig 1) : les coefficients de corrélation entre les valeurs des 2 années pour un même animal varient de 0,79 à 0,89 
Tableau I. Variations des caractéristiques rhéologiques du lait entre la première et la deuxième lactation.

\begin{tabular}{|c|c|c|c|c|}
\hline & \multicolumn{4}{|c|}{ Numéro de lactation } \\
\hline & 1 & 2 & $E T R^{\mathrm{c}}$ & \\
\hline$N$ & 20 & 20 & & \\
\hline \multicolumn{5}{|l|}{ Hiver a } \\
\hline $\mathrm{pH}$ & 6,70 & 6,71 & 0,05 & NS \\
\hline Taux de caséines $(g / l)$ & 25,6 & 24,2 & 1,8 & $\cdot$ \\
\hline Taux butyreux $(g / l)$ & 35,8 & 35,7 & 4,0 & NS \\
\hline Protéines solubles $(g / l)$ & 5,8 & 6,1 & 0,5 & NS \\
\hline Calcium $(g / l)$ & 1,12 & 1,32 & 0,07 & ** \\
\hline Temps de coagulation (min) & 12,1 & 11,6 & 2,5 & NS \\
\hline Temps de raffermissement (min) & 7,6 & 9,0 & 3,9 & NS \\
\hline Fermeté du gel $(\mathrm{mm})$ & 37,3 & 35,0 & 8,8 & NS \\
\hline \multicolumn{5}{|l|}{ Étéb } \\
\hline $\mathrm{pH}$ & 6,71 & 6,73 & 0,05 & NS \\
\hline Taux de caséines $(g / l)$ & 28,5 & 28,2 & 2,1 & NS \\
\hline Taux butyreux $(\mathrm{g} / \mathrm{l})$ & 37,3 & 36,9 & 4,5 & NS \\
\hline Protéines solubles $(\mathrm{g} / \mathrm{l})$ & 5,7 & 6,6 & 0,7 & $* *$ \\
\hline Calcium (g/l) & 1,38 & 1,52 & 0,14 & ** \\
\hline Temps de coagulation (min) & 13,4 & 10,8 & 2,8 & ** \\
\hline Temps de raffermissement (min) & 7,4 & 5,2 & 2,6 & * \\
\hline Fermeté du gel $(\mathrm{mm})$ & 37,7 & 46,1 & 9,6 & * \\
\hline
\end{tabular}

a Moyenne de 3 prélèvements ; ${ }^{b}$ moyenne de 2 prélèvernents ; ${ }^{c}$ écart type résiduel; * $P<0,05 ;{ }^{* *}: P<0,01$.

selon les paramètres et la période (hiver ou été), soit des valeurs semblables à celles obtenues pour le taux de caséines.

\section{DISCUSSION}

Cette étude a mis en évidence une amélioration sensible de l'aptitude à la coagulation du lait entre la première et la seconde lactation, comme cela est parfois rapporté par les producteurs de fromages fermiers (Martin, communication personnelle), et conformément aux travaux de Schaar (1984), mais contrairement à ceux réalisés par Auriol (1961) et Lindström et al (1984) ; mais ces dernières observations ne concernaient que le temps de coagulation, et n'avaient pas été réalisées sur les mêmes animaux au cours de lactations successives. Compte tenu des écarts de taux de caséines du lait au cours de l'hiver, certainement liés à une alimentation énergétique légèrement meilleure chez les primipares, cette différence ne s'exprime qu'au cours de l'été où les taux de caséines sont voisins entre les 2 années. Cet écart ne peut pas être imputé à la méthode de mesure, compte tenu de l'absence de variation des caractéristiques rhéologiques du lait témoin tout au long des 10 séries de mesures effectuées. Par ailleurs, la seconde année, des prélèvements réalisés en même temps sur des vaches en pre- 

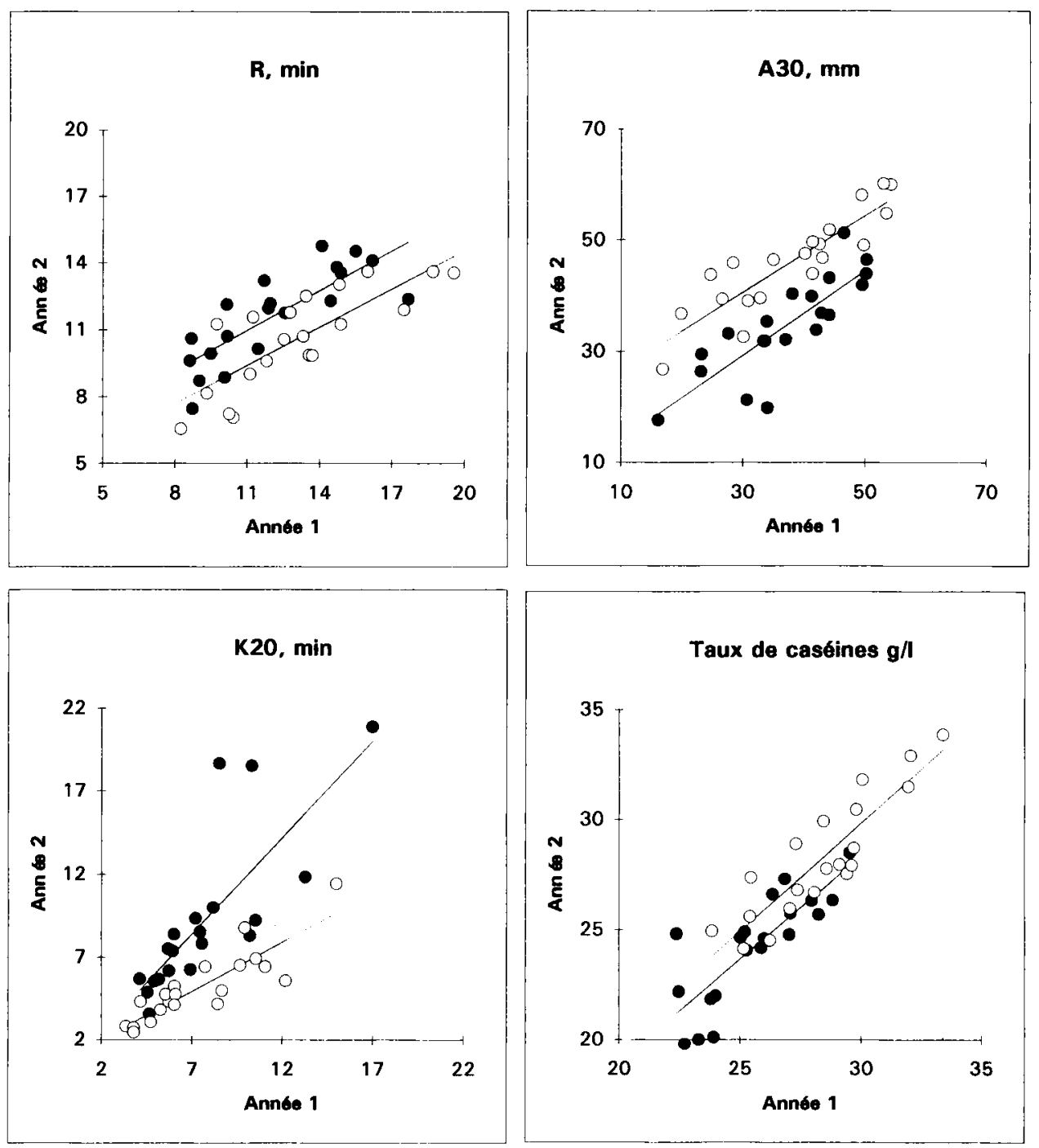

Fig 1. Liaisons entre les paramètres de la coagulation du lait d'une lactation à l'autre chez les mêmes vaches. R : temps de coagulation ; K20 : temps de raffermissement ; A30 : fermeté du gel. • Moyenne des 3 prélèvements hivernaux; o moyenne des 2 prélèvements estivaux.

mière ou en seconde lactation, conduites dans des conditions comparables, ont confirmé l'existence d'un léger écart des caractéristiques rhéologiques du lait, à même taux de caséines, en faveur des vaches en seconde lactation (tableau II). Ces écarts sont liés en partie aux différences de $\mathrm{pH}$ entre les 2 types d'animaux; 
Tableau II. Variations des caractéristiques rhéologiques du lait, mesurées la seconde année sur 20 vaches primipares et 21 vaches en deuxième lactation.

\begin{tabular}{lcccc}
\hline & \multicolumn{4}{c}{ Numéro de lactation } \\
\cline { 2 - 5 } & 1 & 2 & ETR \\
\hline $\mathrm{N}$ & 20 & 21 & & \\
$\mathrm{pH}$ & 6,72 & 6,68 & 0,04 & $*$ \\
Calcium (g/l) & 1,30 & 1,30 & 0,06 & $\mathrm{NS}$ \\
Temps de coagulation (min) & 12,6 & 11,1 & 2,3 & $\mathrm{NS}$ \\
Temps de raffermissement (min) & 9,2 & 7,2 & 2,8 & $*$ \\
Fermeté du gel (mm) & 33,5 & 38,2 & 6,7 & $*$ \\
& & & & \\
\hline
\end{tabular}

Moyennes de 3 prélèvements hivernaux. Valeurs ajustées par analyse de variance pour tenir compte de la race des animaux - Holstein ou Montbéliarde -, du variant de la caséine kappa - AA ou AB -, et du taux de caséines du lait. ${ }^{*} P<0,10$.

en effet, à pH standardisé, les caractéristiques d'aptitude à la coagulation ne présentent plus de différences significatives entre primipares et multipares. Les causes possibles de cet écart sont difficiles à cerner. Elles pourraient être liées à des modifications entre la première et la deuxième lactation: i) de l'effet de certains variants génétiques des protéines du lait, comme cela a été montré sur la composition chimique du lait par $\mathrm{Ng}-\mathrm{K} w a i-H a n g$ et al (1990); ii) de la composition des caséines du lait (augmentation de la part de la caséine kappa dont on connaît l'importance dans la phase de coagulation du lait); iii) de la taille des micelles, dont on sait qu'elle peut varier au cours de la lactation (Holt et al, 1978) ; mais aucun travail expérimental ne permet à notre connaissance de confirmer ces hypothèses; iv) des équilibres salins du lait (Scharr, 1984), et en particulier de sa teneur en calcium. Le temps de coagulation du lait est en effet bien lié au rapport Ca/azote du lait (Mocquot et al, 1954 ; Auriol, 1961), et dans notre étude, le taux de calcium a été nettement supérieur en seconde lactation. Cependant, on considère habituellement que ce taux est très peu variable avec l'âge (Guéguen, 1971), et il est possible que l'écart observé dans cette étude ne soit pas totalement dû à un effet propre de l'âge, mais à des facteurs liés à l'année de mesure, non appréhendés dans cette étude. En effet, d'une part l'augmentation du taux de calcium en seconde lactation a été accompagnée d'une diminution du taux de caséines, contrairement à la relation positive couramment observée entre ces 2 paramètres, et d'autre part, la seconde année, les taux de calcium mesurés sur des vaches en première ou en seconde lactation ont été identiques (tableau II).

\section{RÉFÉRENCES}

Auriol P (1961) Quelques facteurs de variation du temps de coagulation des laits individuels de vaches. Ann Biol Anim Biochim Biophys $1,152-162$

Coulon JB, Chilliard Y, Rémond B (1991) Effets du stade physiologique et de la saison sur la composition chimique du lait de vache et ses caractéristiques technologiques (aptitude à la coagulation, lipolyse). INRA Prod Anim 4, 219-228 
Guéguen L (1971) La composition minérale du lait et son adaptation aux besoins minéraux du jeune. Ann Nutr Alim 25, 335-381

Grosclaude $F$ (1988) Le polymorphisme génétique des principales lactoprotéines bovines. Relations avec la quantité, la composition et les aptitudes fromagères du lait. INRA Prod Anim 1, 5-17

Holt C, Baird L, Muir DD (1978) Natural variations in the average size of bovine casein $\mathrm{mi}$ celles. J Dairy Res 45, 339-352

Lindström UB, Antila V, Syvajarvi J (1984) A note on some genetic and non-genetic factors affecting clotting time of Ayrshire milk. Acta Agric Scand 34, 349-355

Machebœuf D, Coulon JB, D'Hour P (1993) Effect of breed, protein genetic variants and feeding on cows' milk coagulation properties. $J$ Dairy Res 60, 43-54

McMahon DJ, Brown RJ (1982) Evaluation of Formagraph for comparing rennet solutions. J Dairy Sci 65, 1639-1642

Mocquot G, Alais C, Chevalier R (1954) Étude sur les défauts de coagulation du lait par la présure. Ann Tech Agric 1, 1-44

$\mathrm{Ng}$-Kwai-Hang KF, Monardes HG, Hayes JF (1990) Association between genetic polymorphism of milk proteins and production traits during three lactations. J Dairy Sci 73, 34143420

Schaar J (1984) Effects of Kappa-casein genetic variants and lactation number on the renneting properties of individual milks. $J$ Dairy Res $51,397-406$ 